\title{
Another advance in wireless optogenetics
}

Researchers lighten the load and increase the channels for multi-site optogenetics.

Now over a decade old, the field of optogenetics continues to play an important role in understanding neural circuitry, letting researchers control neural activity in animal models by activating light-sensitive proteins with different sources of light. The technology continues to advance, becoming more refined in its capabilities while also less cumbersome for the animal bearing the implanted equipment.

A collaboration between a team of bioengineers in the lab of John Rogers at the University of Illinois and neuroscientists at Washington University School of Medicine in St. Louis has been working towards improving optogenetic technology since their first paper on a wireless system (Science 12, 211-216; 2013). Whereas the initial technology was centered on the brain, they wanted to develop a platform that could function anywhere in the nervous system. In previously published work led by Sung Il Park, they engineered a miniaturized, soft, stretchable system with a micro-LED photostimulation source that could be implanted anywhere under the skin. The microLEDs were smaller than traditionally used optical fibers, improving the precision to target discrete structures. Powered by radio signals (and thus battery-free), the platform was also wireless, cutting the tether that could otherwise interfere with normal animal behavior. After validating the technology in Illinois, neuroscientists under Michael Bruchas and Robert Gereau in St. Louis used the system to investigate pain pathways through peripheral nerves and the spinal cord (Nat. Biotechnol. 33, 1280-1286; 2015).

The collaboration is back to the brain in a new paper published in the Proceedings of the National Academy of Sciences USA (113, E8169-E8177). Park, now an assistant professor at Texas A\&M University, explains that despite its improvements over other techniques, the previous platform was still limited to stimulation of a single target with a single wavelength of light. That limitation leaves more complex circuits that are controlled by different neurons or from different regions, such as those behind contrasting behavioral responses like preference and aversion, out of reach. Testing opposing responses would require two different animals, far from an ideal solution.

The latest advancement, which he considers version 1.5, "allows for independent control of multiple light sources in a single platform." Modifying the original stretchable implant, the new device presented includes a multichannel antenna and two microLEDs. The radio frequency energy harvester, which also powers the system, can receive different signal frequencies from a transmitter and then active the different channels accordingly. For example in the paper, a $2.3 \mathrm{GHz}$ signal activated a green microLED via the first channel, while the second channel was tuned to $2.7 \mathrm{GHz}$ to turn on a blue light. Additionally, the system features a biodegradable needle, allowing the microLEDs to be implanted into deep tissues without causing damage to extract the needle.

Over in the neuroscience labs at Washington University, the platform was put to the test in vivo for two complex behavioral applications: sleep arousal, modulated deep in the brain by the locus coeruleus, and preference/aversion responses involving the nucleus accumbens. In the first case, Cre-expressing mice sleeping normally in their home cages were remotely awoken by photostimulation from the implant; control littermates slept on undisturbed. The second application took advantage of the multichannel design to individually stimulate neurons in the dorsal and ventral sides of the nucleus accumbens, separated by less than a millimeter in the brain, as the animals freely moved about a conditioning chamber. The dorsal side influences preference, the ventral side aversion; independently stimulating

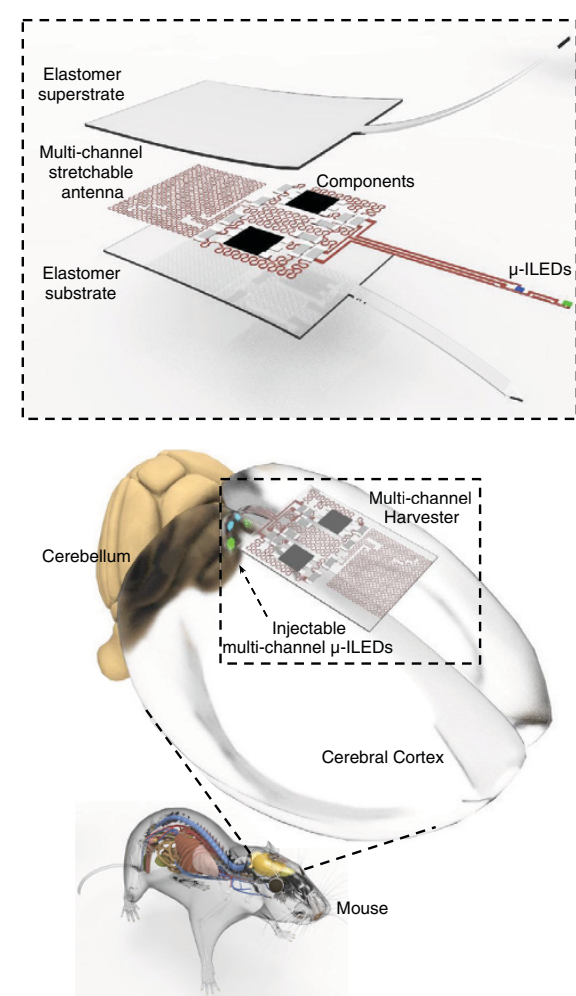

Diagrams of the multichannel, soft wireless system and its use in the brain. Image adapted from Proc. Natl. Acad. Sci. USA 113, E8169-E8177; 2016.

the different areas altered the animal's behavior accordingly.

With up to eight channels available, Park envisions version 2.0 as a totally closedloop system, able to record neural activity, analyze the data in real-time, and then modulate the signaling pattern. In the future, he is interested in studying pain signaling via the spinal cord with the device to tease out the transition from acute pain to chronic. Bruchas' lab studies stress, an application in which he finds a minimally invasive, wireless system particularly useful to keep anthropogenic sources down while attempting to assess how circuits are functioning to mediate behavior.

Ellen P. Neff 